\title{
Bell's Theorem without Inequalities and without Alignments
}

\author{
Adán Cabelld* \\ Departamento de Fúsica Aplicada II, Universidad de Sevilla, 41012 Sevilla, Spain
}

(Dated: November 1, 2018)

\begin{abstract}
A proof of Bell's theorem without inequalities is presented which exhibits three remarkable properties: (a) reduced local states are immune to collective decoherence; (b) distant local setups do not need to be aligned, since the required perfect correlations are achieved for any local rotation of the local setups; (c) local measurements require only individual measurements on the qubits. Indeed, it is shown that this proof is essentially the only one which fulfils (a), (b), and (c).
\end{abstract}

PACS numbers: 03.65.Ud, 03.65.Ta, 03.67.Hk

The proofs of Bell's theorem without inequalities [1, 2, $[3,4,[5,6]$ are based on the existence, predicted by quantum mechanics, of certain perfect correlations between results of spacelike separated measurements. However, perfect (or almost perfect) correlations between results of distant measurements are difficult to achieve in real experiments 7]. Besides "practical" reasons such as imperfect preparations or imperfect detector efficiencies [8], there are two main difficulties for obtaining perfect correlations between distant measurements. The first is decoherence, i.e., the fact that reduced quantum states suffer unwanted couplings with the environment during their flight to the distant regions. The second is the need for a perfect alignment between the source emitting entangled states and the setups of the distant measurements 9]. For the proofs $[1,2,3,4,5,6]$, any imperfection in the required alignments leads to the disappearance of the required perfect correlations.

In this Letter it is shown that both difficulties can be overcome. For this purpose, a proof of Bell's theorem without inequalities for two observers is introduced. This proof exhibits three remarkable properties: (a) reduced local states are immune to collective decoherence, (b) distant local setups do not need to be aligned, since the required perfect correlations are achieved for any local rotation of the local setups, and (c) local measurements require only individual measurements on the qubits. Property (c) is very useful for practical purposes because, as shown below, in order to fulfil (a) and (b), each of the two local subsystems should consist of at least four qubits. Indeed, it will be shown that the proposed proof is essentially the only one which fulfils (a), (b), and (c).

We shall assume that, during their flight, the reduced quantum states suffer a particularly relevant form of decoherence known as collective decoherence 10, 11, 12, 13. Collective decoherence occurs whenever the spatial/temporal separation between the qubits is small relative to the correlation length/time of the environment. In this scenario, the environment couples with the qubits without distinguishing between them and, as a consequence of the interaction, all qubits undergo the same unknown but unitary evolution. Therefore, a state $|\psi\rangle$ of $N$ qubits is immune to collective decoherence if and only if $|\psi\rangle$ is invariant under the tensor product of $N$ equal unitary operators, i.e., $U^{\otimes}|\psi\rangle=|\psi\rangle$ [10, 11, 12, 13]. States of this type exist for $N$ even and the smallest nontrivial subspace spanned by such states occurs for $N=4$ qubits [10, 11, 12, 13].

Strategies to establish a common direction or Cartesian frame between distant observers to any desired accuracy have attracted much attention in recent times 14, 15, 16, 17, 18]. These papers have drawn attention to two points relevant to our discussion. First, a shared common reference frame so that distant observers may prepare and measure spin components relative to it should not be considered a free preexisting element in any communication scenario but should instead be considered an expensive resource. Second, if such a resource is not given, establishing a perfect alignment between local reference frames requires an infinite amount of communication. This has motivated the interest in methods for the distribution of quantum information between parties who do not share any reference frame [19, 20].

The proposed proof of Bell's theorem without inequalities is as follows. Consider a source emitting systems of eight qubits prepared in the state

$$
|\eta\rangle=\left(\left|\phi_{0} \phi_{0}\right\rangle+\sqrt{3}\left|\phi_{0} \phi_{1}\right\rangle+\sqrt{3}\left|\phi_{1} \phi_{0}\right\rangle\right) / \sqrt{7},
$$

where $\left|\phi_{0}\right\rangle$ and $\left|\phi_{1}\right\rangle$ are the two singlet states obtained adding up four spin- $\frac{1}{2}$ momenta,

$$
\begin{aligned}
\left|\phi_{0}\right\rangle= & \frac{1}{2}(|0101\rangle-|0110\rangle-|1001\rangle+|1010\rangle), \\
\left|\phi_{1}\right\rangle= & \frac{1}{2 \sqrt{3}}(2|0011\rangle-|0101\rangle-|0110\rangle-|1001\rangle \\
& -|1010\rangle+2|1100\rangle) .
\end{aligned}
$$

These states were introduced by Kempe et al. in the context of decoherence-free fault-tolerant universal quantum computation [13]. Let us suppose that the first four qubits prepared in $|\eta\rangle$ fly to Alice and the second four qubits fly to a distant observer, Bob. On her/his four qubits, each observer randomly chooses to measure either $F$ or $G$, defined as

$$
\begin{aligned}
& F=-\left|\phi_{0}\right\rangle\left\langle\phi_{0}|+| \phi_{1}\right\rangle\left\langle\phi_{1}\right|, \\
& G=-\left|\psi_{0}\right\rangle\left\langle\psi_{0}|+| \psi_{1}\right\rangle\left\langle\psi_{1}\right|,
\end{aligned}
$$


where $\left|\psi_{0}\right\rangle$ and $\left|\psi_{1}\right\rangle$ are obtained, respectively, from $\left|\phi_{0}\right\rangle$ and $\left|\phi_{1}\right\rangle$, by permuting qubits 2 and 3, i.e.,

$$
\begin{aligned}
\left|\psi_{0}\right\rangle= & \frac{1}{2}(|0011\rangle-|0110\rangle-|1001\rangle+|1100\rangle) \\
= & \frac{1}{2}\left(\left|\phi_{0}\right\rangle+\sqrt{3}\left|\phi_{1}\right\rangle\right) \\
\left|\psi_{1}\right\rangle= & \frac{1}{2 \sqrt{3}}(-|0011\rangle+2|0101\rangle-|0110\rangle-|1001\rangle \\
& +2|1010\rangle-|1100\rangle) \\
= & \frac{1}{2}\left(\sqrt{3}\left|\phi_{0}\right\rangle-\left|\phi_{1}\right\rangle\right) .
\end{aligned}
$$

The observable $F(G)$ has three possible outcomes: -1 , corresponding to $\left|\phi_{0}\right\rangle\left(\left|\psi_{0}\right\rangle\right), 1$ corresponding to $\left|\phi_{1}\right\rangle$ $\left(\left|\psi_{1}\right\rangle\right)$, and 0 , which never occurs because the local subsystems have total spin zero. Measuring $F$ is thus equivalent to distinguishing with certainty between $\left|\phi_{0}\right\rangle$ and $\left|\phi_{1}\right\rangle$ with a single test on the four qubits, and measuring $G$ is equivalent to distinguishing with certainty between $\left|\psi_{0}\right\rangle$ and $\left|\psi_{1}\right\rangle$. Alice's measurements on qubits 1 to 4 are assumed to be spacelike separated from Bob's measurements on qubits 5 to 8 .

The state $|\eta\rangle$ can also be expressed as

$$
\begin{aligned}
|\eta\rangle= & \left(4\left|\phi_{0} \psi_{0}\right\rangle+\sqrt{3}\left|\phi_{1} \psi_{0}\right\rangle+3\left|\phi_{1} \psi_{1}\right\rangle\right) / 2 \sqrt{7} \\
= & \left(4\left|\psi_{0} \phi_{0}\right\rangle+\sqrt{3}\left|\psi_{0} \phi_{1}\right\rangle+3\left|\psi_{1} \phi_{1}\right\rangle\right) / 2 \sqrt{7} \\
= & \left(7\left|\psi_{0} \psi_{0}\right\rangle+3 \sqrt{3}\left|\psi_{0} \psi_{1}\right\rangle+3 \sqrt{3}\left|\psi_{1} \psi_{0}\right\rangle\right. \\
& \left.-3\left|\psi_{1} \psi_{1}\right\rangle\right) / 4 \sqrt{7} .
\end{aligned}
$$

Moreover, since $\left|\phi_{0}\right\rangle,\left|\phi_{1}\right\rangle,\left|\psi_{0}\right\rangle$, and $\left|\psi_{1}\right\rangle$ are invariant under the tensor product of four equal unitary operators, then they are invariant under local rotations. Therefore, expressions (11) and (8)-(10) remain unchanged after local rotations. Consequently, if $R_{A}$ and $\mathcal{R}_{A}\left(R_{B}\right.$ and $\left.\mathcal{R}_{B}\right)$ are rotations of Alice's (Bob's) setups for measuring, respectively, $F$ and $G$ relative to the reference frame of the source then, in the state $|\eta\rangle$, for any rotations $R_{A}, \mathcal{R}_{A}$, $R_{B}$, and $\mathcal{R}_{B}$,

$$
\begin{aligned}
P\left(R_{A} F=1, R_{B} F=1\right) & =0, \\
P\left(R_{A} F=1 \mid \mathcal{R}_{B} G=1\right) & =1, \\
P\left(R_{B} F=1 \mid \mathcal{R}_{A} G=1\right) & =1, \\
P\left(\mathcal{R}_{A} G=1, \mathcal{R}_{B} G=1\right) & =\frac{9}{112}
\end{aligned}
$$

where $P\left(R_{A} F=1, R_{B} F=1\right)$ is the joint probability that both Alice and Bob obtain the outcome 1 when both perform experiment $F$ (or any experiment consisting on independently rotating their setups for measuring $F)$, and $P\left(R_{A} F=1 \mid \mathcal{R}_{B} G=1\right)$ is the probability that Alice obtains the outcome 1 when she performs experiment $F$ (or any experiment consisting on rotating her setup for measuring $F$ ), conditioned to Bob obtaining the outcome 1 when he performs experiment $G$ (or any experiment consisting on rotating his setup for measuring $G$ ).

From property (14), if both Alice and Bob choose the setup for measuring $G$, then in $8 \%$ of the events the outcome is 1 in both cases. This is true even if Alice applies any rotation $\mathcal{R}_{A}$ to her setup and Bob applies any rotation $\mathcal{R}_{B}$ to his setup.

From property (13), if Alice measures $G$ and obtains the outcome 1, then she can predict with certainty that, if Bob measures $F$, he will obtain 1. According to Einstein, Podolsky, and Rosen (EPR), this fact must be interpreted as sufficient evidence that there is a local "element of reality" in Bob's qubits determining this outcome 21]. Moreover, EPR reasoning seems to be even more inescapable in our example, since Alice's prediction with certainty is valid even if Alice applies any rotation $\mathcal{R}_{A}$ to her setup for measuring $G$ and Bob applies any rotation $R_{B}$ to his setup for measuring $F$.

Analogously, from property (12), if Bob measures $G$ (or $\mathcal{R}_{A} G$ ) and obtains 1 , then he can predict with certainty that, if Alice measures $F$ (or $R_{A} F$ ), she will obtain 1. Again, according to EPR, there must be a local element of reality in Alice's qubits determining this outcome.

Therefore, assuming EPR's point of view, for at least $8 \%$ of the systems prepared in the state $|\eta\rangle$, there must be two joint local elements of reality: one for $\mathrm{Al}-$ ice's qubits, corresponding to $R_{A} F=1$, and one for Bob's qubits, corresponding to $R_{B} F=1$. However, this inference is in contradiction with property (11), which states that the joint probability of obtaining the outcomes $R_{A} F=1$ and $R_{B} F=1$ is zero. This provides a simple and powerful proof that the concept of element of reality, as defined by EPR, is incompatible with quantum mechanics, even if the predictions with certainty are valid not only for a particular alignment of the distant setup but for any possible alignment.

The logical argument in the previous proof is similar to the one in Hardy's [4]. However, this proof exhibits some remarkable features:

(a) Partial states are immune to collective decoherence.-While the reduced states required in previous proofs of Bell's theorem without inequalities [1, 2, 3, 4, 5, 6] are destroyed under collective decoherence, the reduced states used in the proof above are immune to collective decoherence. This can be seen by expressing the reduced density matrix describing both local states as

$$
\rho=\left[(7+\sqrt{13})\left|\chi^{+}\right\rangle\left\langle\chi^{+}|+(7-\sqrt{13})| \chi^{-}\right\rangle\left\langle\chi^{-}\right|\right] / 14,
$$

where

$$
\left|\chi^{ \pm}\right\rangle=\left[(1 \pm \sqrt{13})\left|\phi_{0}\right\rangle+2 \sqrt{3}\left|\phi_{1}\right\rangle\right] / \sqrt{26 \pm 2 \sqrt{13}}
$$

Since $\left|\phi_{0}\right\rangle$ and $\left|\phi_{1}\right\rangle$ are invariant under any tensor product of four equal unitary operators, then any incoherent 
superposition of them, such as $\rho$, is also invariant and therefore is immune to collective decoherence.

(b) Distant local setups do not need to be aligned, since the required perfect correlations are achieved for any local rotation of the setups.-This property is derived from the fact that measuring the local observable $F(G)$ is equivalent to distinguishing with certainty between the orthogonal states $\left|\phi_{0}\right\rangle$ and $\left|\phi_{1}\right\rangle\left(\left|\psi_{0}\right\rangle\right.$ and $\left.\left|\psi_{1}\right\rangle\right)$, and that both states (and thus any other two states obtained by permuting qubits) are invariant under any tensor product of four equal unitary operators, and thus under any local rotation of the setup for measuring $F(G)$.

(c) Local observables can be measured by means of tests on individual qubits. - A practical advantage and a very remarkable property of this proof is that measuring $F$ or $G$ does not require collective measurements on two or more qubits but instead a single test on each of the four qubits. Measuring $F$ is equivalent to distinguishing between $\left|\phi_{0}\right\rangle$ and $\left|\phi_{1}\right\rangle$ with a single test. Remarkably, the only two orthogonal states invariant under any tensor product of four equal unitary operators that can be reliably distinguished by fixed (as opposed to conditioned, as those in 22]) measurements on the four individual qubits are $\left|\phi_{0}\right\rangle$ and $\left|\phi_{1}\right\rangle$ and those obtained from them by permuting qubits (such as $\left|\psi_{0}\right\rangle$ and $\left|\psi_{1}\right\rangle$ ). To prove this, let us consider two orthogonal states invariant under any tensor product of four equal unitary operators,

$$
\begin{aligned}
|\psi\rangle & =\cos \omega\left|\phi_{0}\right\rangle+\sin \omega\left|\phi_{1}\right\rangle, \\
\left|\psi^{\perp}\right\rangle & =\sin \omega\left|\phi_{0}\right\rangle-\cos \omega\left|\phi_{1}\right\rangle .
\end{aligned}
$$

States $|\psi\rangle$ and $\left|\psi^{\perp}\right\rangle$ are reliably distinguishable by fixed measurements on the four individual qubits if and only if there is an orthogonal local basis $\left\{\left|0_{a}\right\rangle \otimes\left|0_{b}\right\rangle \otimes\left|0_{c}\right\rangle \otimes\right.$ $\left.\left|0_{d}\right\rangle, \ldots,\left|1_{a}\right\rangle \otimes\left|1_{b}\right\rangle \otimes\left|1_{c}\right\rangle \otimes\left|1_{d}\right\rangle\right\}$ in which, for all $j$ such that the $j$ component of $|\psi\rangle\left(\left|\psi^{\perp}\right\rangle\right)$ is not zero, then the $j$ component of $\left|\psi^{\perp}\right\rangle(|\psi\rangle)$ is necessarily zero. Since $|\psi\rangle$ and $\left|\psi^{\perp}\right\rangle$ are invariant under any tensor product of four equal unitary operators, we can restrict our attention, without losing generality, to the case of spin measurements in the $x-z$ plane. Then, orthogonal local basis are composed by states of the form

$$
\begin{aligned}
& \left|0_{a}\right\rangle=\cos \theta_{a}|0\rangle+\sin \theta_{a}|1\rangle, \\
& \left|1_{a}\right\rangle=\sin \theta_{a}|0\rangle-\cos \theta_{a}|1\rangle .
\end{aligned}
$$

If $|\psi\rangle$ and $\left|\psi^{\perp}\right\rangle$ are distinguishable in a basis of this type, then, when expressed in such a basis, the first component of one of the two states must be zero. After some algebra, it can be seen that a necessary condition for the first component of $|\psi\rangle$ to be zero is

$$
\begin{aligned}
\cot \omega= & \frac{1}{\sqrt{3}} \csc \left(\theta_{a}-\theta_{b}\right) \csc \left(\theta_{c}-\theta_{d}\right) \\
& \times\left[\cos \left(\theta_{a}+\theta_{b}-\theta_{c}-\theta_{d}\right)\right. \\
& \left.-\cos \left(\theta_{a}-\theta_{b}\right) \cos \left(\theta_{c}-\theta_{d}\right)\right] .
\end{aligned}
$$

This condition makes zero both the first and last component of $|\psi\rangle$. Components two to eight can be expressed in terms of $\omega, \theta_{a}, \theta_{b}, \theta_{c}$, and $\theta_{d}$. Components nine to 15 are identical to components two to eight but in reverse order and with opposite signs. If $|\psi\rangle$ and $\left|\psi^{\perp}\right\rangle$ are distinguishable in a basis of this type, then more components should also be zero. The important point is that the cost of obtaining more zeroes is to restrict the possible values of $\omega$. It can be checked that any way to make more than four zeroes (one of the two orthogonal states must have more than four zeroes) requires

$$
\omega=n \pi / 6
$$

(with $n$ integer). We therefore conclude that the only two four-qubit states invariant under any tensor product of four equal unitary operators that are reliably distinguishable by fixed measurements on individual qubits are those with $\omega=n \pi / 6$ (with $n$ integer). Note, however, that these states are precisely those obtained from $\left|\phi_{0}\right\rangle$ and $\left|\phi_{1}\right\rangle$ by permuting qubits.

We shall now show that, to distinguish with certainty between $\left|\phi_{0}\right\rangle$ and $\left|\phi_{1}\right\rangle$, it is enough to measure the spin component of the first two qubits along the same direction and the spin component of the other two qubits along a perpendicular direction. This can be seen by resorting to the invariance under any tensor product of four equal unitary operators and expressing these states in the basis of eigenstates of $\sigma_{z 1} \otimes \sigma_{z 2} \otimes \sigma_{x 3} \otimes \sigma_{x 4}$,

$$
\begin{aligned}
\left|\phi_{0}\right\rangle= & \frac{1}{2}(-|01 \overline{0} \overline{1}\rangle+|01 \overline{1} \overline{0}\rangle+|10 \overline{0} \overline{1}\rangle-|10 \overline{1} \overline{0}\rangle), \\
\left|\phi_{1}\right\rangle= & \frac{1}{2 \sqrt{3}}(|00 \overline{0} \overline{0}\rangle-|00 \overline{0} \overline{1}\rangle-|00 \overline{1} \overline{0}\rangle+|00 \overline{1} \overline{1}\rangle \\
& -|01 \overline{0} \overline{0}\rangle+|01 \overline{1} \overline{1}\rangle-|10 \overline{0} \overline{0}\rangle+|10 \overline{1} \overline{1}\rangle \\
& +|11 \overline{0} \overline{0}\rangle+|11 \overline{0} \overline{1}\rangle+|11 \overline{1} \overline{0}\rangle+|11 \overline{1} \overline{1}\rangle),
\end{aligned}
$$

where $\sigma_{z}|0\rangle=|0\rangle, \sigma_{z}|1\rangle=-|1\rangle, \sigma_{x}|\overline{0}\rangle=|\overline{0}\rangle, \sigma_{x}|\overline{1}\rangle=$ $-|\overline{1}\rangle[|\overline{0}\rangle=(|0\rangle+|1\rangle) / \sqrt{2}$ and $|\overline{1}\rangle=(|0\rangle-|1\rangle) / \sqrt{2}]$. According to (23) and (24), if the measurements on the individual qubits are $\sigma_{z 1}, \sigma_{z 2}, \sigma_{x 3}, \sigma_{x 4}$ (or any rotation thereof), then, among the 16 possible outcomes, four occur (with equal probability) only in the state $\left|\phi_{0}\right\rangle$, and the other twelve occur (with equal probability) only in the state $\left|\phi_{1}\right\rangle$ (this has been experimentally demonstrated in [20]). Therefore, to measure $F(G)$, it is enough to measure the spin component of qubits 1 and 2 (1 and 3 ) along the same direction and the spin component of the other two qubits along a perpendicular direction.

(d) Contradiction is nearly optimal.- The fact that, except for permutations of the qubits, the only two orthogonal four-qubit states invariant under any tensor product of four equal unitary operators that can be reliably distinguished by fixed measurements on individual qubits are $\left|\phi_{0}\right\rangle$ and $\left|\phi_{1}\right\rangle$ means that the only local observables whose eigenvectors are invariant under any tensor 
product of four equal unitary operators and can be reliably distinguished by fixed measurements on the four individual qubits are, precisely, $F$ and $G$. This enormously restricts the possible proofs of Bell's theorem without inequalities which satisfy (a), (b), and (c). By checking every possible combination of states and observables, it can be seen that the proof presented here exhibits the maximum probability, $\frac{9}{112} \approx 0.08$, for a Hardy-like contradiction satisfying (a), (b), and (c). Without requirement (c), it can be proven (as in [4]) that the maximum probability for a Hardy-like contradiction is $[(\sqrt{5}-1) / 2]^{5} \approx 0.09$. Therefore, the maximum probability for a Hardy-like contradiction satisfying (a), (b), and (c) is close to the optimal probability without these requirements.

In summary, some recent methods of decoherence-free fault-tolerant universal quantum computation have been used to illustrate that some difficulties that were previously assumed to be inherent to any proof of Bell's theorem without inequalities can indeed be overcome.

On the experimental side, while the four-qubit states $\left|\phi_{0}\right\rangle$ and $\left|\phi_{1}\right\rangle$ have already been prepared and their immunity to collective decoherence and invariance under local rotations tested in a laboratory [20], preparing entangled superpositions thereof, such as $|\eta\rangle$, is a significant goal for future research.

I thank M. Bourennane and H. Weinfurter for useful discussions, J. L. Cereceda and N. D. Mermin for their comments on the manuscript, and the Spanish Ministerio de Ciencia y Tecnología Project No. BFM2002-02815, the Junta de Andalucía Project No. FQM-239, and the European Science Foundation (Quantum Information Theory and Quantum Computation Short Scientific Visit Grants Program) for support.

* Electronic address: adan@us.es

[1] D.M. Greenberger, M.A. Horne, and A. Zeilinger, in Bell's Theorem, Quantum Theory, and Conceptions of the Universe, edited by M. Kafatos (Kluwer, Dordrecht, 1989), p. 69.

[2] N.D. Mermin, Phys. Rev. Lett. 65, 3373 (1990).

[3] L. Hardy, Phys. Rev. Lett. 68, 2981 (1992).

[4] L. Hardy, Phys. Rev. Lett. 71, 1665 (1993).

[5] A. Cabello, Phys. Rev. Lett. 86, 1911 (2001).

[6] A. Cabello, Phys. Rev. Lett. 87, 010403 (2001).

[7] For experimental results see, for instance, D. Boschi, S. Branca, F. De Martini, and L. Hardy, Phys. Rev. Lett.
79, 2755 (1997)

[8] The detection efficiency is not a fundamental problem in the sense that there are quantum systems for which the efficiency of the detectors is almost perfect. While, for polarization-entangled photons, detection efficiency is just about $5 \%$ [see, for instance, G. Weihs, T. Jennewein, C. Simon, H. Weinfurter, and A. Zeilinger, Phys. Rev. Lett. 81, 5039 (1998)], similar experiments with trapped ions have achieved nearly perfect detection efficiency [see, for instance, M.A. Rowe, D. Kielpinski, V. Meyer, C.A. Sackett, W.M. Itano, C. Monroe, and D.J. Wineland, Nature (London) 409, 791 (2001)].

[9] For instance, Yuval Ne'eman defended that the answer to the puzzle posed by Bell's theorem was to be found in the implicit assumption that the detectors were aligned. Ne'eman apparently believed that the two detectors were connected through the space-time affine connection of general relativity [N.D. Mermin (private communication)].

[10] L.-M. Duan and G.-C. Guo, Phys. Rev. Lett. 79, 1953 (1997).

[11] P. Zanardi and M. Rasetti, Phys. Rev. Lett. 79, 3306 (1997).

[12] D.A. Lidar, I.L. Chuang, and K.B. Whaley, Phys. Rev. Lett. 81, 2594 (1998).

[13] J. Kempe, D. Bacon, D.A. Lidar, and K.B. Whaley, Phys. Rev. A 63, 042307 (2001).

[14] A. Peres and P.F. Scudo, Phys. Rev. Lett. 86, 4160 (2001).

[15] A. Peres and P.F. Scudo, Phys. Rev. Lett. 87, 167901 (2001).

[16] E. Bagan, M. Baig, A. Brey, R. Muñoz-Tapia, and R. Tarrach, Phys. Rev. A 63, 052309 (2001).

[17] E. Bagan, M. Baig, and R. Muñoz-Tapia, Phys. Rev. A 64, 022305 (2001).

[18] E. Bagan, M. Baig, and R. Muñoz-Tapia, Phys. Rev. Lett. 87, 257903 (2001).

[19] S.D. Bartlett, T. Rudolph, and R.W. Spekkens, Phys. Rev. Lett. 91, 027901 (2003). The possibility of testing Bell's inequalities in the absence of a shared reference frame was pointed out in this reference. Testing Bell's inequalities, however, does not require perfect alignments.

[20] M. Bourennane, M. Eibl, S. Gaertner, C. Kurtsiefer, A. Cabello, and H. Weinfurter, quant-ph/0309041 [Phys. Rev. Lett. (to be published)].

[21] According to EPR, if Alice can predict, with certainty and without in any way disturbing Bob's qubits, the value of a physical quantity of Bob's qubits, then there exists a local element of physical reality corresponding to this physical quantity [see A. Einstein, B. Podolsky, and N. Rosen, Phys. Rev. 47, 777 (1935)].

[22] J. Walgate, A.J. Short, L. Hardy, and V. Vedral, Phys. Rev. Lett. 85, 4972 (2000). 